\title{
A New Centralized Multicast Wavelength Allocation Algorithm in Optical Fiber Network
}

\author{
${ }^{*}$ Rafed Sabbar Abbas, ${ }^{2}$ Hamed Saffa Al-Raweshidy \\ Wireless network and Communication Centre \\ School of Engineering and Design, Brunel University \\ London, U.K. \\ E-mail: ${ }^{1}$ Rafedsabbar.abbas@brunel.ac.uk, ${ }^{2}$ Hamed.Al-Raweshidy@brunel.ac.uk
}

\begin{abstract}
Wavelength assignment is a widely research object and especially in optical networks. The centralized algorithm has been proposed in this paper and there are two scenarios for our algorithm. The main effect and improvement in the algorithm is to save network capacity by decreasing network capacity after each wavelength assignment. The term network capacity and network capacity decreasing are well investigated and analyzed. There are also mathematical equations for them have been formed and created. By our knowledge, this is the first time the equations are created. The performance of our network is mainly depending on the perfect complete binary, ternary and quaternary. The idea is rare investigated in perfect complete network, while the other works usually study random networks. The work in this paper is concentrated on centralized algorithm wherever the future work will compare the distributed algorithms also. The research is found the blocking probability, capacity decreasing and the time delay for different networks and compared with different algorithms. The results show the clear improvement in our algorithm. These algorithms are suitable for using in passive optical networks, because we did not use any active devices in our scenarios.
\end{abstract}

Keywords: wavelength allocation, capacity decreasing, binary and ternary trees, passive optical network, centralized technology.

\author{
* Corresponding address: \\ Rafed Sabbar Abbas, \\ Wireless Network and Communication Centre, School of Engineering and Design, Brunel University, London, \\ United Kingdom \\ E-mail: $\underline{\text { rafedsabba.abbas@brunel.ac.uk }}$ \\ Tell: $+44(0) 7410256970$
}

\section{Introduction}

The optical network technology has the key for the current and future revolution in the communication. In the past few years, the wavelength-division - multiplexing (WDM) networks have been the strongest candidate for the future high requirement to provide enormous available network. Dense wavelength-division-multiplexing (DWDM) can provide a hundreds of channels (wavelengths) for each individual optical fiber. There are dual and simplex fiber cables. The dual core system allows data to be transferred in bi-directional, as opposed to simplex fiber cables, which typically only propagate data in one direction. The bidirectional communication is achieved over single-mode simplex fiber using multiple wavelengths when the Wavelength Division Multiplexing (WDM) systems are utilized. Clearly, the use of WDM has the potential of improving the performance of modern lightwave systems by a factor of more than one million [1]. Wavelength collision is caused if optical signals from two inputs are forwarded to the same output using the same wavelength. The wavelength conversion (WC) technologies are used in the WDM network with different schemes. The wavelength conversion (WC) technologies are sorted by the conversion degree (d) as has been analyzed by [2]. The dynamic wavelength assignment (DWA) has been implemented in different algorithms, in which it has different schemes and computations. The centralized and distributed schemes is represented the (DWA) algorithms. In [3]-[6] the centralized scheme has been implemented. In [3], they investigate the problem of wavelength assignment in wavelength reusable multi-carrier distributed (WRMD) wavelength-division-multiplexing (WDM) ring networks. In conventional WDM ring networks, each edge node (EN) has its own light sources, and optical channels, called light-paths, are established by using optical carriers generated from laser diodes (LDs) at the source EN. In this network, light-paths between source and destination ENs are established by using carriers generated from a centralized multi-carrier light source. In [4] work, a centralized network controller is used, 
estimating the impact of the physical impairments on the quality of a light-path before provisioning it can cause a significant delay.

Quality of service aware wavelength assignment algorithms are proposed that consider both bit-error rate (BER) and latency constraints. The experimental result [5] of Dynamic Impairment Constraint Optical Networking (DICONET) proposal for centralized and distributed control plane integration schemes have been proposed with all-optical mesh networks. In this paper [6], they present an advanced multicast algorithm based on node heterogeneity. The experiment results show this algorithm can reduce message redundancy and multicast delay to a greater extent. In [7] they have proposed a priority search technique to route multicast sessions in a WDM mesh network. The simulation result shows that the proposed algorithm increases the throughput of communication in a delay constrained application by reducing rate of request blocking in a sparse-split constrained finite wavelength WDM mesh network. The multi and uni-cast have been investigated in the (DWA) using various algorithms. Multicast technology can be defined one-to-many or many-to-many data transfer. There are source and destinations or sources and destinations. Multicast technology is implemented by using a multicast source to avoid sending a separate copy of the data to each receiver. Therefore, the data is transmitted only once. The aim of this work is to increase network capacity, minimizing time delay reduce blocking probability. It is investigated because of the importance of optical networks. This work limitation is about centralized computation in broadcast technology in wired networks.

\section{Multicast Technology}

The optical fiber tree networks is represented by a directed topology tree graph and it is $\mathrm{T}(\mathrm{N}, \mathrm{E})$, where $\mathrm{N}$ is the number of vertices (nodes) and $\mathrm{E}$ the number of direct edges (links) between any two nodes (parent and its child). A multicast request can be represented by a set of $\mathrm{T}(\mathrm{v}, \mathrm{d})$, where $\mathrm{v}$ is the source (root node) and $\mathrm{d}$ is the number of destinations (leaf nodes). A multicast tree $\mathrm{Y}\left(N_{Y}, E_{Y}\right)$ is shaped when each multicast request is made. As can be noticed in the tree topology, the number of nodes is $\mathrm{N}$ and the number of links in any tree network is $\mathrm{N}-1$. Let $\mathrm{W}$ represent the number of wavelengths available in network and let make the wavelengths available in the tree are $\mathrm{W}=\{\lambda 1, \lambda 2, \lambda 3 \ldots \lambda \mathrm{n}\}$. Each link in the tree has some available wavelength(s) that can be used to transfer data. The sub-trees formatted by the multicast are represented as $\mathrm{Y}=\{\mathrm{Y} 1, \mathrm{Y} 2, \mathrm{Y} 3 \ldots \mathrm{Yn}\}$. They are different in various algorithms, because they assign different wavelengths in their computation. The algorithms and their performance will be analyzed later on this paper.

\section{Tree Networks Analyzing}

Tree networks have various structures depend on if they are symmetrical or not. The symmetrical tree networks can be designed as a binary, ternary, quaternary, octal trees, etc. That sequence depend on the number of the children nodes to each parent node. The unary tree can be studied by the uni-cast only because there is just on child for each parent, in another word there is one source and one destination. The binary, ternary and quaternary are investigated in this research. The network capacity is calculated and a specific equation for each type of these networks has been proposed. The network capacity is assumed in two situations: when the source is just the root node, the other when all parent nodes are data sources.

A tree $\mathrm{T}$ with $\mathrm{h}$ levels is complete if all levels except possibly the last are completely full, and the last level has all its nodes to the left side. The work in this paper is related to the complete trees.

Network capacity of number of calls is one of the most important factors in any telecommunication network is a mean purpose for many companies and a lot of researches. Therefore, the work in this paper has investigated this term and compared it in different situations. In this step, the network capacity decreasing is described how it occurs in a multicast tree network after each wavelength assignment, and from that the network capacity decreasing can be minimized as much as possible.

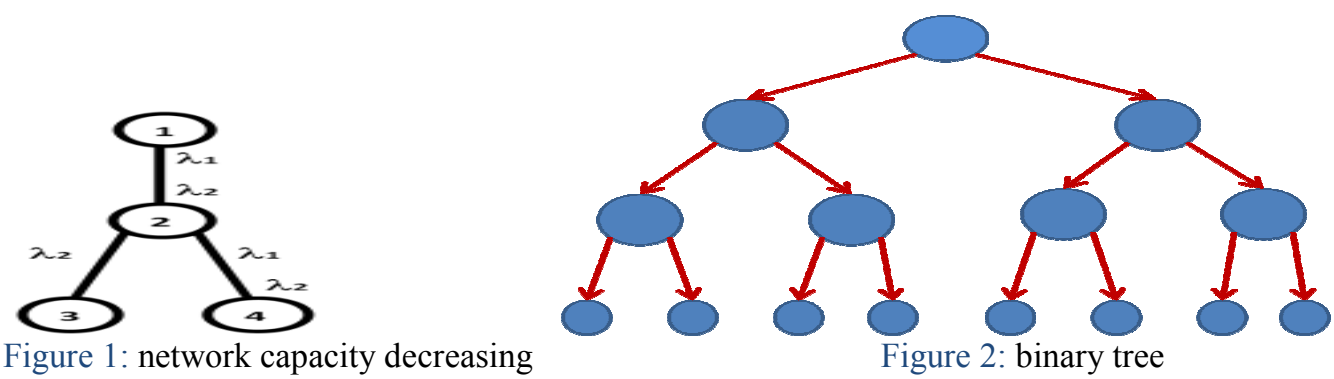


International Journal of

Soft Computing And Software Engineering (JSCSE)

e-ISSN: 2251-7545

Vol.2,No.11, 2012

Published online: Nov 25, 2012
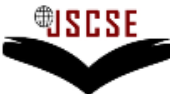

DOI: $10.7321 /$ jscse.v2.n11.2

\subsection{Network Capacity}

Network capacity is represent the number of calls that any network can serve. If a network has served the peak value of calls, then any call coming will be blocked or is put on hold until any other present call is turned off. Therefore, this search is coming to increase network capacity by using special algorithm. The algorithm will be analyzed later on this paper, but the network capacity is described and calculated in this step.

In the network in figure (1), there are availability for the calls $\{(1,2),(2,3),(2,4),(1,3)$ and $(1,4)\}$. There are five calls for each wavelength available in this network as downstream calls. If both downstream and upstream calls are assumed, the number of calls available in this network will be ten. But we assume the downstream calls in our investigation.

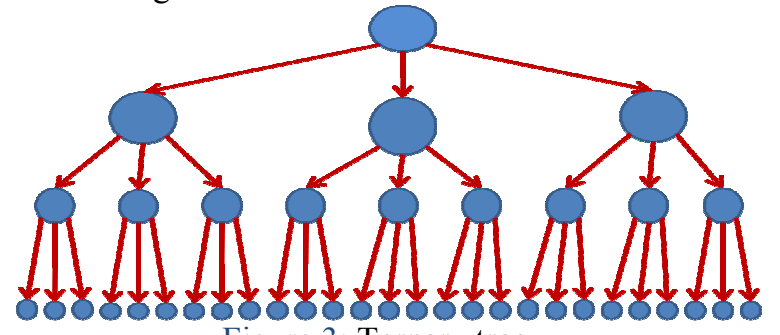

Figure 3: Ternary tree

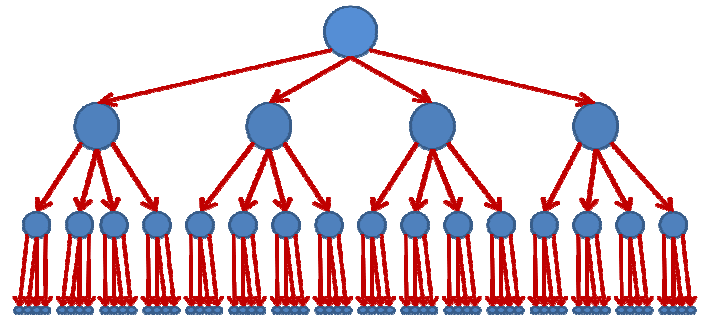

Figure 4: Quaternary tree

The networks capacities, in this work, are depended on the symmetric design (binary, ternary and quaternary). In the binary tree, shown in figure (2), two calls can be made from the root node to its children. There are four calls also from the root node to the four nodes in the level two, and also four calls from the two nodes in level one to the four nodes in level two. Eight calls also available, in this network, from root node, its two children and the four nodes in level two to the eight nodes in the level three. The total of the calls in this network are thirty four calls. They are depending on the level of the network.

If the network has one level $(\mathrm{h}=1)$, number of calls $=2$.

While in the network consist of two levels $(\mathrm{h}=2)$, number of calls $=2+8=10$.

In network of three levels $(\mathrm{h}=3)$, number of calls $=2+8+24=34$.

Mathematically, we have created our new equation and it is formulated as below:

$\left(2^{1}+2^{2}+2^{3}+\ldots\right) \times h=2+8+24+\ldots$.

$\frac{2}{2}=1, \frac{8}{4}=2, \frac{24}{8}=3$

That means they depend on their depth (level), therefore, the equation can be written as:

$\left.\left[\left(2^{1} \times h\right)+\left(2^{2} \times h\right)+\left(2^{3} \times h\right)+\ldots.\right)\right]=2+8+24+\ldots$.

$\left.\left[\left(2^{1} \times 1\right)+\left(2^{2} \times 2\right)+\left(2^{3} \times 3\right)+\ldots ..\right)\right]=2+8+24+\ldots .$.

Where $h$ is depth of the network

Then, the binary network capacity is formulated as:

network capacity $\left.=\left[\left(2^{1} \times h\right)+\left(2^{2} \times h\right)+\left(2^{3} \times h\right)+\ldots.\right)\right]=\sum_{n=1}^{h} 2^{n} \times n(1)$

The equation for ternary network capacity (Fig (3)) is written as:

ternary network capacity $=\sum_{n=1}^{h} 3^{n} \times n$

The equation for ternary network capacity (Fig (4)) is written as:

quaternary network capacity $=\sum_{n=1}^{h} 4^{n} \times n$

For all other networks, Fig (5), the equation can be generally formulated as:

network capacity $=\sum_{n=1}^{h} m^{n} \times n$

Where, $(m)$ is the arranging of the network as shown in figure (5). 


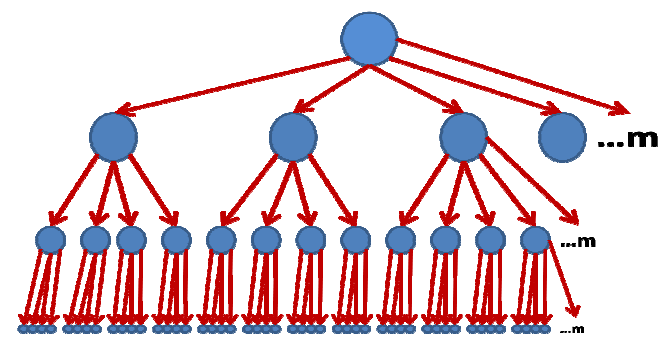

Figure 5: Network with $m$ arranging

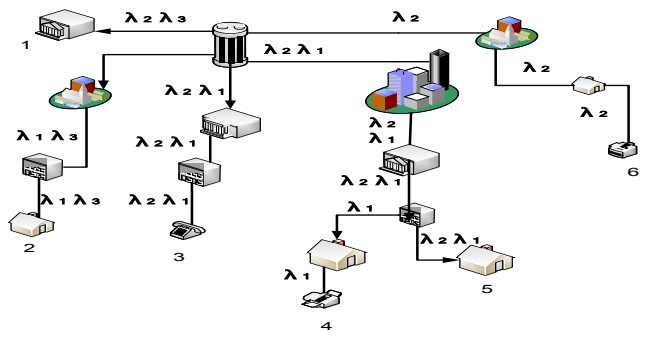

Figure 6: Network showing performance for SCG and DCG

\subsection{Capacity Decreasing in Multicast [8]}

The tree T with $h$ levels and $n$ number of nodes, each node has two children as shown in fig (1). If there is a call requested to destination (4). If $(\lambda 1)$ is assigned for the call $\{1,4\}$, the network capacity decreasing will be $\{(1$, $2),(2,4)$ and $(1,4)\}$, and result is $(3)$ call availabilities are blocked, as shown in fig $(1)$. Whereas, if $(\lambda 2)$ is assigned for the call $\{1,4\}$, network capacity decreasing will be $\{(1,2),(2,4),(1,4)$ and $(1,3)\}$, and result is (4) calls will be blocked. Therefore $(\lambda 1)$ is assigned instead of $(\lambda 2)$ to decrease network capacity by (3) calls instead of (4).

\subsection{Multicast Algorithms}

Multicast algorithm is the steps that have to be followed to assign a wavelength to broadcast information and calls to different destination. Multicast technology can be defined one-to-many or many-to-many data transfer. There are source and destinations or sources and destinations. Multicast technology is implemented by using a multicast source to avoid sending a separate copy of the data to each receiver. Therefore, the data is transmitted only once.

\subsubsection{Highest Number of Destinations algorithm (HND)}

Multicast technology in all-optical network gives us ability to send the same data to multiple-destinations. Using (HND) algorithm is to minimize network capacity decreasing after each wavelength multicast. Performance of our algorithm is to calculate the number of destinations $\left(D_{k}\right)$, the wavelength $\lambda_{k}$ can reach. The number of destinations $\left(D_{k}\right)$ for each wavelength is only computed once in the centralized static highest number of destinations (CSHND) algorithm. While in centralized dynamic highest number of destinations (CDHND), the number of destination $\left(D_{k A}\right)$ for each wavelength is calculated after any wavelength assignment. $\left(C_{k}\right)$ is the cost of assigning wavelength $\left(\lambda_{k}\right)$ to reach these $\left(D_{k}\right)$ destinations.

We have designed three tree networks explaining the (HND) algorithm performance compared with greedy algorithms in [9] and how they decrease network capacity. The HND algorithm has been assumed as centralized algorithm in this step to be equitable when it is compared with the centralized greedy algorithms.

The centralized technologies mainly depend on the wavelength continuity constraint and assume that the wavelength is available along the path from the source to the destinations. The wavelength continuity constraint is usually used when the wavelength conversion is required to be reduced, because the wavelength continuity constraint does not need any wavelength conversion through its work. Therefore, it has been widely used in the passive optical networks.

\subsubsection{Performance}

Here is the analysis of the network capacity decreasing and the different between the centralized and distributed technology. The explanation has been made by using mathematic analyzing. There is also a mathematic calculation of the number of links and paths, and how they are blocked after each call. The tree network has many sub trees,

$\mathrm{T}=(\mathrm{S} 1, \mathrm{~S} 2, \mathrm{~S} 3, \mathrm{~S} 4 \ldots . . \mathrm{Sn})$, where $n$ is the number of wavelengths in the main tree. Network capacity is as defined in equation (2). Where ( $\mathrm{L}$ is the links in the network and $\mathrm{P}$ is number of paths.

We can specify sub-trees related to its wavelength assignment cost and these will be:

$S_{\emptyset 1}, S_{\emptyset 2}, S_{\emptyset 3}, S_{\emptyset 4}, \ldots S_{\emptyset n}$

Where $S_{\emptyset 1} \leq S_{\emptyset 2} \leq S_{\emptyset 3} \leq S_{\emptyset 4} \leq \cdots S_{\emptyset n}$

The network capacity decreasing can be calculated in centralized method by find:

$S_{\emptyset 1} \cap S_{\emptyset 2} \cap S_{\emptyset 3} \cap S_{\emptyset 4} \cap \ldots S_{\emptyset n}$ 
International Journal of

Soft Computing And Software Engineering (JSCSE)

e-ISSN: 2251-7545

Vol.2,No.11, 2012

Published online: Nov 25, 2012

DOI: 10.7321/jscse.v2.n11.2

If we specify every sub-tree to its paths to destinations, the sub-trees will be:

$S_{\emptyset 1}=\left(S_{P 1}, S_{P 2}, S_{P 3} \ldots S_{P p}\right), \quad S_{\emptyset 2}=\left(S_{P 1}, S_{P 2} \ldots S_{P p}\right), \ldots S_{\emptyset n}$

(3)

Where $\mathrm{p}$ is number of destinations and $\mathrm{P}$ is function for number of links.

$P_{1}=\left(L_{1}, L_{2}, L_{3}, \ldots L_{l}\right), P_{2}=\left(L_{1}, L_{2}, L_{3}, \ldots L_{l}\right)$

Therefore its work will increase network capacity with each intersected path between the sub-trees. Network capacity decrease $\left(C_{d}\right)$ for the algorithms computation will be:

$$
C d=C p-\left[S_{\emptyset 1}+\left(S_{\emptyset 2}-\left(S_{\varnothing 2} \cap S_{\emptyset 1}\right)\right)+\left(S_{\emptyset 3}-\left(S_{\varnothing 3} \cap S_{\emptyset 1}\right)-\left(S_{\emptyset 3} \cap S_{\emptyset 2}\right)\right) \ldots S_{\emptyset n}\right]
$$

Whereas the network distributed method will account the number of links. Its work will increase network capacity with each intersected link between the sub-trees and therefore we have to specify sub-trees according to their links:

$$
\left\{S_{\varnothing 1}=\left(L_{1}, L_{2}, L_{3} \ldots L_{l}\right), S_{\varnothing 2}\left(L_{1}, L_{2}, L_{3} \ldots L_{l}\right), \ldots S_{\varnothing n}\right\}
$$

Where, $l$ is number of links.

Therefore network capacity decreasing $\left(C_{d}\right)$ in a network using distributed algorithm, will be the same equation (eq. (5)) for the centralized algorithm but depend on the number of links in the network.

\subsubsection{HND works better than SCG and DCG}

In Fig (6), if we have calls requested to nodes $(1,2,3,4,5,6)$, (CSHND) accounts $\left(D_{1}=4, D_{2}=4\right.$ and $\left.D_{3}=2\right)$ and therefore it connects $\lambda_{1}$ to nodes $(2,3,4$ and 5$)$ and $\lambda_{2}$ to nodes (1 and 6). (CDHND) connects $\lambda_{1}$ to nodes (2, 3, 4 and 5) and it accounts $\left(D_{2}=2\right.$ and $\left.D_{3}=1\right)$, therefore it chooses $\lambda_{2}$ to nodes (1 and 6). (SCG) will account $\emptyset_{k}$ for every $\left(\lambda_{k}\right), \emptyset_{1}=21 / 4=5.25, \emptyset_{2}=18 / 4=4.5, \emptyset_{3}=6 / 2=3$. SCG select $\lambda_{3}$ to reach nodes $(1$ and 2$), \lambda_{2}$ to reach nodes $(3,5$ and 6$)$ and finally use $\lambda_{1}$ to reach node (4). (DCG) choose $\lambda_{3}$ to reach nodes (1 and 2) and then compute $\emptyset_{1}$ and $\emptyset_{2}$ and they are 4.67 and 5 therefore choose $\lambda_{1}$ to reach nodes $\left(3,4\right.$ and 5) and $\lambda_{2}$ to reach node (6). In this situation the (SCG) decreases network capacity by 39, (CSHND) and (CDHND) reduce by 36 and (DCG) reduces it by 38 .

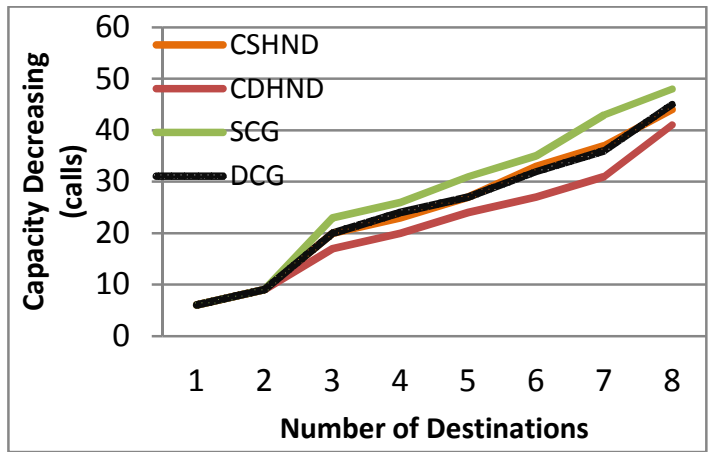

Fig (7) Capacity Decreasing in Binary network

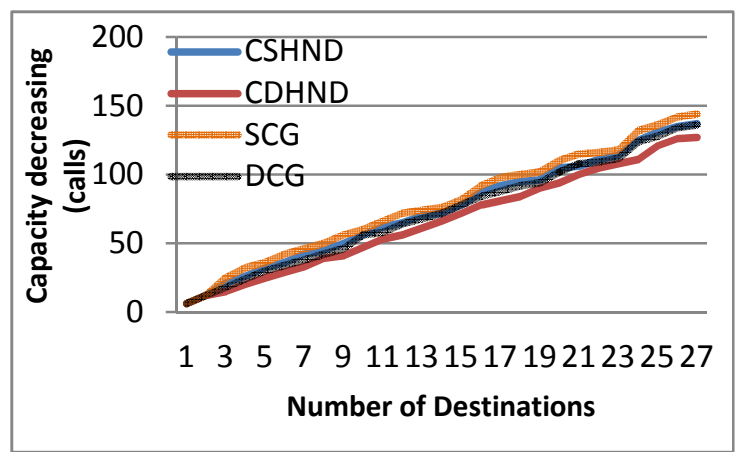

Fig (8) Capacity Decreasing in Ternary network

\section{Results}

By using numerical calculation for the network capacity decreasing test as shown in Fig (6 and 7) the improvement is clear among the HND algorithm scenarios and the greedy algorithm (CG) scenarios. In the first graph the simulation is about a binary tree with three levels, four wavelengths, eight destinations, fifteen nodes and two wavelengths available to each destination. While the simulation in Fig (7) showing the result for a ternary network with three levels and consist of forty nodes, twenty seven destinations, four wavelengths and 
two wavelengths available to each destination. The performance relating network capacity for the DCG and CSHND is about the same. The CDHND scenario has the best performance among all other technologies.

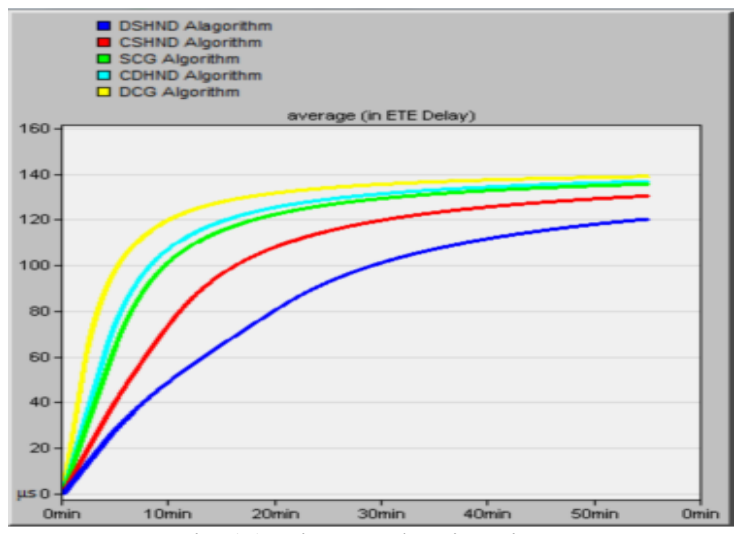

Fig (9) Time Delay in Binary Tree

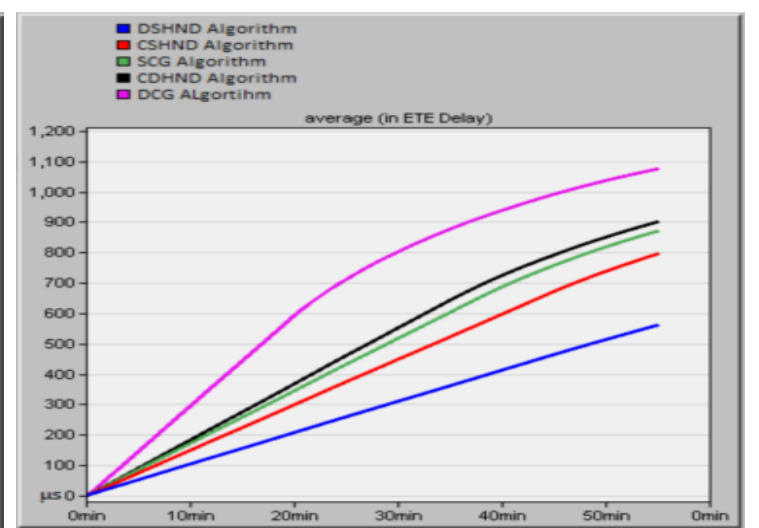

Fig (10) Time Delay in Ternary Tree

By using Opnet software to find the time delay for the different scenarios and algorithms, the results is as shown in Fig ( 8 and 9). The time delay is found for a binary network Fig (8) with three levels. The parameters for the network are ten kilometer square, four wavelengths, ten kilobit packet size, packet interval time is four, simulation time is fifty five minutes and there are two wavelengths available for each destination. The network size is shown in Fig (2). The simulation for a ternary network, Fig (9), is the same parameters of the binary network but the just difference is in the network size as shown in Fig (3). The time delay for our algorithm with the static scenario (CSHND) has the smallest value. The performance of the CDHND and SCG is very near. The distributed scenario of our proposed algorithm has less value of the all centralized algorithms and scenarios. Therefore, distributed scenario will be our future work.

\section{Conclusion}

The centralized algorithm is proposed and investigated in this paper. There are two scenarios are assumed for our algorithm. Passive optical network is high worth in telecommunication networks because of the energy saving and green technology. Therefore, our algorithm is proved to be suitable for the passive optical networks. The network capacity and the number of calls in any communication network are extremely important and our algorithm has improved network capacity and it saved the network capacity decreasing after each assignment of a wavelength. The network capacity decreasing is compared with a greedy algorithm and the result proved of the improvement of our algorithm. The time delay is also simulated to find the performance of the algorithms and that showed the less time delay is for our algorithm with the static scenario. The network trees have been chosen with an order coordinator and they were not chosen randomly as the most of other researches. The network capacity is formulated in mathematical equations by our work and these equations are published in the first time as our knowledge.

\section{Future Work}

The future work is to investigate distributed scheme and compare it with our centralized scheme. It is also to investigate wavelength switch and how it can be improved by minimizing wavelength conversion range, number of converters and number of splitters to minimize complication of switching and its cost.

\section{References:}

[1] Kai Chang, "Fiber-Optic Communication Systems", A John Wiley \& Sons, INC., USA, 2010.

[2] Abbas, R.S.; Al-Raweshidy, H.; , "New multicast switch-based multicast wavelength allocation algorithm to increase network capacity," Future Communication Networks (ICFCN), 2012 International Conference on , vol., no., pp.164-169, 2-5 April 2012.

[3] Keri, M.; Oki, E.; Matsuura, M.; , "Wavelength Assignment in Multi-Carrier Distributed Optical Ring Networks With Wavelength Reuse," Optical Communications and Networking, IEEE/OSA Journal of , vol.3, no.4, pp.281-289, April 2011. 
[4] Jun He; Brandt-Pearce, M.; Subramaniam, S.; , "QoS-Aware Wavelength Assignment With BER and Latency Constraints for All-Optical Networks," Lightwave Technology, Journal of , vol.27, no.5, pp.462-474, March1, 2009.

[5] Azodolmolky, S.; Perelló, J.; Angelou, M.; Agraz, F.; Velasco, L.; Spadaro, S.; Pointurier, Y.; Francescon, A.; Saradhi, C.V.; Kokkinos, P.; Varvarigos, E.; Al Zahr, S.; Gagnaire, M.; Gunkel, M.; Klonidis, D.; Tomkos, I.; , "Experimental Demonstration of an Impairment Aware Network Planning and Operation Tool for Transparent/Translucent Optical Networks," Lightwave Technology, Journal of , vol.29, no.4, pp.439-448, Feb.15, 2011.

[6] Yan Wang; Zhao-Kui Li; , "A multicast advanced algorithm based on node heterogeneity for P2P MMOG," Future Computer and Communication (ICFCC), 2010 2nd International Conference on, vol.2, no., pp.V2-165-V2-168, 21-24 May 2010.

[7] Barat, S.; Kumar, A.; Pradhan, A.K.; De, T.; , "A light-forest approach for QoS multicasting in WDM networks," Industrial and Information Systems (ICIIS), 2012 7th IEEE International Conference on, vol., no., pp.1-6, 6-9 Aug. 2012.

[8] Sabbar Abbas, Rafed; Al-Raweshidy, Hamed Saffa; Radhi, Nazar; Alabdulkreem, Eatedal; , "New multicast switch and algorithm to increase network capacity," International Conference on Telecommunications (ICT), 2012 19th, vol., no., pp.1-6, 23-25 April 2012.

[9] Jianping Wang; Biao Chen; Uma, R.N.; , "Dynamic wavelength assignment for multicast in all-optical WDM networks to maximize the network capacity," Selected Areas in Communications, IEEE Journal on , vol.21, no.8, pp. 1274- 1284, Oct. 2003. 